\title{
Differentiability v.s. Convexity for Complementarity Functions
}

\author{
Chien-Hao Huang \\ Department of Mathematics \\ National Taiwan Normal University \\ Taipei 11677, Taiwan \\ E-mail: qqnick0719@ntnu.edu.tw \\ Jein-Shan Chen ${ }^{1}$ \\ Department of Mathematics \\ National Taiwan Normal University \\ Taipei 11677, Taiwan \\ E-mail: jschen@math.ntnu.edu.tw \\ Juan Enrique Martinez-Legaz ${ }^{2}$ \\ Departament d'Economia i d'Història Econòmica \\ Universitat Autònoma de Barcelona \\ 08193 Bellaterra, Spain \\ and \\ Barcelona Graduate School of Mathematics (BGSMath), Barcelona, Spain \\ E-mail: JuanEnrique.Martinez.Legaz@uab.cat
}

March 24, 2015

(1st revised on April 20, 2015)

(2nd revised on October 21, 2015)

\begin{abstract}
It is known that complementarity functions play an important role in dealing with complementarity problems. The most well known complementarity problem is the symmetric cone complementarity problem (SCCP) which includes nonlinear complementarity problem (NCP), semidefinite complementarity problem (SDCP), and second-order cone complementarity problem (SOCCP) as special cases. Moreover, there is also socalled generalized complementarity problem (GCP) in infinite dimensional space. Among the existing NCP-functions, it was observed that there are no differentiable and convex

\footnotetext{
${ }^{1}$ Corresponding author. The author's work is supported by Ministry of Science and Technology, Taiwan.

${ }^{2}$ The work of this author has been supported by the MINECO of Spain, Grant MTM2014-59179C2-2-P, and under Australian Research Council's Discovery Projects funding scheme (project number DP140103213). He is affiliated to MOVE (Markets, Organizations and Votes in Economics).
} 
NCP-functions. In particular, Miri and Effati [20] show that convexity and differentiability cannot hold simultaneously for an NCP-function. In this paper, we further establish that such result also holds for general complementarity functions associated with the GCP.

Keywords: Complementarity functions, NCP-functions, second-order cone, closed convex cone.

\section{Introduction}

The complementarity problem arises from the KKT conditions of an optimization problem. Formally, it seeks to find an element $x$ such that

$$
x \succeq_{\mathcal{K}} 0, \quad F(x) \succeq_{\mathcal{K}} 0, \quad\langle x, F(x)\rangle=0,
$$

where $\mathcal{K}$ is usually a symmetric cone $[11], \succeq_{\mathcal{K}}$ is the partial order associated with $\mathcal{K}$, and $\langle\cdot, \cdot\rangle$ is an appropriate inner product. When $\mathcal{K}$ is the nonnegative orthant, the above problem (1) reduces to the well known nonlinear complementarity problem (NCP for short) which consists in finding a point $x \in \mathbb{R}^{n}$ such that

$$
x \geq 0, \quad F(x) \geq 0, \quad\langle x, F(x)\rangle=0,
$$

where $\langle\cdot, \cdot\rangle$ is the Euclidean inner product and $F=\left(F_{1}, \ldots, F_{n}\right)^{T}$ is a map from $\mathbb{R}^{n}$ to $\mathbb{R}^{n}$. NCPs have wide applicability in the fields of economics, engineering, and operations research, see $[9,10,13,20]$ and references therein. When $\mathcal{K}$ represents a positive semidefinite cone $\mathcal{S}_{+}^{n}$, the complementarity problem (1) reduces to a semidefinite complementarity problem (SDCP for short). When $\mathcal{K}$ is the second-order cone (SOC) whose definition will be introduced later, the complementarity problem (1) is the second-order cone complementarity problem (SOCCP for short). All the above special cases can be unified as symmetric cone complementarity problem (SCCP) under Euclidean Jordan algebra.

Besides the symmetric cone complementarity problem which is endowed in a finite dimensional space, we further consider the generalized complementarity problem (GCP for short) in infinite dimensional space. More specifically, let $(X,\|\cdot\|)$ denote a real Banach space, $X^{*}$ represent its dual space, we consider a cone $K$ which is solid (i.e., int $K \neq \emptyset)$ closed convex in $X$. Note that its dual cone $K^{+}$is defined as

$$
K^{+}=\left\{x^{*} \in X^{*}:\left\langle x, x^{*}\right\rangle \geq 0, \forall x \in K\right\} .
$$

In contrast to the aforementioned symmetric cone, $K$ is not self-dual in general. Let $\langle\cdot, \cdot\rangle: X \times X^{*} \rightarrow \mathbb{R}$ be the canonical bilinear pairing and $F: X \rightarrow X^{*}$. The generalized complementarity problem (GCP) is to find an element $x \in X$ such that

$$
x \in K, \quad F(x) \in K^{+}, \quad\langle x, F(x)\rangle=0 .
$$


The GCP was originally proposed by Karmardian in 1971, see [17]. For more details regarding GCP including solution methods, properties, and applications, please refer to the textbook [15].

To deal with various complementarity problems, the so-called complementarity functions ( $C$-functions) play crucial roles in designing solution methods. In the setting of NCP, the complementarity function is abbreviated as NCP-function, which is denoted by $\phi: \mathbb{R}^{2} \rightarrow \mathbb{R}$ and defined as

$$
\phi(a, b)=0 \quad \Longleftrightarrow \quad a, b \geq 0, a b=0
$$

During the past four decades, approximately thirty NCP-functions have been proposed, see [12] for a survey. Among the existing NCP-functions, it is observed that none of them is both convex and differentiable. In fact, Miri and Effati [21] show that there is no pseudoconvex NCP-function, which implies that the convexity and differentiability cannot hold simultaneously for a NCP function. The proof is based on the following lemmas.

Lemma 1.1. ([12, Corollary 2]) Let $\phi: \mathbb{R}^{2} \rightarrow \mathbb{R}$ be a convex NCP-function. Then, for any $a, b>0, \phi(a, b)<0$.

Lemma 1.2. ([1, Theorem 3.2, Chapter 3]) Let $f: \mathbb{R}^{n} \rightarrow \mathbb{R}$ be a differentiable function. Then, $f$ is convex if and only if for any $x, y \in \mathbb{R}^{n}$,

$$
f(y) \geq f(x)+\langle\nabla f(x), y-x\rangle .
$$

Lemma 1.3. ([21, Lemma 3.1]) Let $\phi: \mathbb{R}^{2} \rightarrow \mathbb{R}$ be an NCP-function. If the first order partial derivatives of $\phi$ exist at the origin, then $\nabla \phi(0,0)=(0,0)^{T}$.

With Lemmas 1.1-1.3, it can be shown that an NCP-function is never convex and differentiable simultaneously. We state this result in Theorem 1.1.

Theorem 1.1. ([21, Corollary 3.1]) Every convex NCP-function is non-differentiable.

Proof. Assume that there exists a differentiable convex NCP-function $\phi: \mathbb{R}^{2} \rightarrow \mathbb{R}$. By Lemma 1.2 and Lemma 1.3, for any $a, b>0$, we have $\phi(a, b) \geq \phi(0,0)+\langle\nabla \phi(0,0),(a, b)\rangle=$ 0, which contradicts Lemma 1.1.

In the setting of SCCP, let $\mathbb{A}=(\mathbb{V},\langle\cdot, \cdot\rangle, \circ)$ denote an $n$-dimensional Euclidean Jordan algebra and $\mathcal{K}$ be the symmetric cone in $\mathbb{V}$. We call $\phi: \mathbb{V} \times \mathbb{V} \rightarrow \mathbb{V}$ a complementarity function ( $C$-function) associated with SCCP if

$$
\phi(x, y)=0 \quad \Longleftrightarrow \quad x \in \mathcal{K}, \quad y \in \mathcal{K}, \quad x \circ y=0 .
$$


Scholars in the field of optimization are interested in this class of functions and the induced merit functions in the sense that these functions help develop algorithms for the symmetric cone complementarity problem and the symmetric cone programming, see $[18,22,24]$ and references therein. As for the GCP setting, a function $\Phi: X \times X^{*} \rightarrow Y$ is called a complementarity function associated with the GCP if

$$
\Phi\left(x, x^{*}\right)=0 \quad \Longleftrightarrow x \in K, \quad x^{*} \in K^{+},\left\langle x, x^{*}\right\rangle=0
$$

where $X, Y$ are Banach spaces.

In this paper, we extend the fact that NCP-functions cannot be simultaneously convex and differentiable to general complementarity functions associated with the general GCP. The key idea is exploiting the concept of cone convexity. In addition, the concept about cone pseudoconvexity is needed, too. To close this section, we recall the definition of cone convexity, which will be used in the main result. Let $X, Y$ be two real Banach spaces and $L$ be a pointed (i.e., $L \cap(-L)=\{0\}$ ) closed convex cone in $Y$.

(a) For $x, y \in Y$ such that $x-y \in L$, we say $x \geq_{L} y\left(\right.$ or $\left.y \leq_{L} x\right)$.

(b) For $x, y \in Y$ such that $x-y \in L \backslash\{0\}$, we say $x>_{L} y\left(\right.$ or $\left.y<_{L} x\right)$. By $x \ngtr_{L} y$ $\left(y \nless_{L} x\right.$ ) we denote the negation of $x>_{L} y$ (respectively, $y<_{L} x$ ).

(c) A mapping $f: X \rightarrow Y$ is said to be $L$-convex if for any $v, w \in X$ and $\lambda \in(0,1)$, one has

$$
f((1-\lambda) v+\lambda w) \leq_{L}(1-\lambda) f(v)+\lambda f(w) .
$$

In particular, if $X=Y=\mathbb{R}^{n}, L=\mathcal{K}^{n}$ is the second-order cone in $\mathbb{R}^{n}$ (also known as the Lorentz cone), which is defined by

$$
\mathcal{K}^{n}=\left\{x=\left(x_{1}, x_{2}\right) \in \mathbb{R} \times \mathbb{R}^{n-1} \mid\left\|x_{2}\right\| \leq x_{1}\right\},
$$

where $\|\cdot\|$ is the Euclidean norm, then the $L$-convexity reduces to the $\mathcal{K}^{n}$-convexity (SOC-convexity), see $[2,5,6]$ for more details.

\section{The main result}

In this section, we show that the differentiability and convexity cannot be held simultaneously for complementarity functions associated with the GCP. Recall that the GCP given as in (2) is to find an element in $X$ such that

$$
x \in K, \quad F(x) \in K^{+}, \quad\langle x, F(x)\rangle=0 .
$$

Here, we require $K$ solid (i.e., int $K \neq \emptyset$ ) closed convex cone in $X$, and $K^{+}$is defined as

$$
K^{+}=\left\{x^{*}:\left\langle x, x^{*}\right\rangle \geq 0, \forall x \in K\right\} .
$$


To achieve the main result for GCP case, we also need some technical lemmas and the concepts about cone-convexity and cone-pseudoconvexity.

Lemma 2.1. Let $(X,\|\cdot\|)$ be a real Banach space and $K$ be a solid closed convex cone in $X$. Then, we have $K-K=X$.

Proof. Let $x \in X$, and pick $k \in \operatorname{int} K$. Then, there exists $t>0$ such that $k+t x \in K$, which yields

$$
x=\frac{1}{t}(k+t x)-\frac{1}{t} k \in K-K .
$$

Thus, the proof is complete.

We assume that $K^{+}$is solid, too. (In connection with this assumption, see $[8$, Theorem 3.6 and Corollary 3.8-3.9].) Hence, by Lemma 2.1, one has

$$
K^{+}-K^{+}=X^{*} \text {. }
$$

Lemma 2.2. Let $f: X \rightarrow Y$ be a Fréchet differentiable mapping and $D f(x)$ denote the derivative of $f$ at $x$. Then, $f$ is $L$-convex if and only if for any $\bar{v}, v \in X$ one has

$$
f(v) \geq_{L} f(\bar{v})+D f(\bar{v})(v-\bar{v}) .
$$

Proof. For any $v, w \in X$ and $\lambda \in(0,1)$, denote $v_{\lambda}=(1-\lambda) v+\lambda w$.

"£" Note that from the assumption, we know

$$
f(v) \geq_{L} f\left(v_{\lambda}\right)+D f\left(v_{\lambda}\right)\left(v-v_{\lambda}\right)=f\left(v_{\lambda}\right)+\lambda D f\left(v_{\lambda}\right)(v-w)
$$

and

$$
f(w) \geq_{L} f\left(v_{\lambda}\right)+D f\left(v_{\lambda}\right)\left(w-v_{\lambda}\right)=f\left(v_{\lambda}\right)+(1-\lambda) D f\left(v_{\lambda}\right)(w-v) .
$$

Adding these two inequalities after multiplying them by $1-\lambda$ and $\lambda$, respectively, we obtain

$$
(1-\lambda) f(v)+\lambda f(w) \geq_{L} f\left(v_{\lambda}\right)
$$

which says $f$ is $L$-convex.

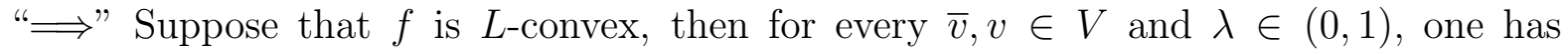
$f((1-\lambda) \bar{v}+\lambda v) \leq_{L}(1-\lambda) f(\bar{v})+\lambda f(v)$. Thus,

$$
\begin{aligned}
f(v)-f(\bar{v})-\frac{f(\bar{v}+\lambda(v-\bar{v}))-f(\bar{v})}{\lambda} & =\frac{(1-\lambda) f(\bar{v})+\lambda f(v)-f((1-\lambda) \bar{v}+\lambda v)}{\lambda} \\
& \in L .
\end{aligned}
$$

Letting $\lambda \rightarrow 0$, we obtain that $f(v)-f(\bar{v})-D f(\bar{v})(v-\bar{v}) \in L$. Then, the proof is complete. 
A Fréchet differentiable mapping $f: X \rightarrow Y$ is said to be $L$-pseudoconvex if the following implications hold true:

$$
\begin{array}{clc}
D f(\bar{v})(v-\bar{v}) \geq_{L} 0 & \Longrightarrow & f(v) \geq_{L} f(\bar{v}), \\
f(v)<_{L} f(\bar{v}) & \Longrightarrow & D f(\bar{v})(v-\bar{v})<_{L} 0 .
\end{array}
$$

By Lemma 2.2, every differentiable $L$-convex function is $L$-pseudoconvex. Moreover, for real valued functions, $L$-pseudoconvexity reduces to the classical notion of pseudoconvexity.

Lemma 2.3. Let $\Phi: X \times X^{*} \rightarrow Y$ be L-pseudoconvex and a $C$-function associated with the GCP. Then, $\Phi\left(x, x^{*}\right) \ngtr_{L} 0$ for any $x \in K$ and $x^{*} \in K^{+}$.

Proof. If we had $\Phi\left(x, x^{*}\right)>_{L} 0$, since $\Phi(2 x, 0)=0$ the second implication in the definition of $L$-pseudoconvexity would yield $D \Phi\left(x, x^{*}\right)\left(x,-x^{*}\right)<_{L} 0$. Similarly, from the equality $\Phi\left(0,2 x^{*}\right)=0$ we would obtain $D \Phi\left(x, x^{*}\right)\left(-x, x^{*}\right)<_{L} 0$, which is a contradiction, since $D \Phi\left(x, x^{*}\right)\left(-x, x^{*}\right)=-D \Phi\left(x, x^{*}\right)\left(x,-x^{*}\right)$.

Lemma 2.4. Let $\Phi: X \times X^{*} \rightarrow Y$ be a $C$-function for the GCP. If $\Phi$ is differentiable at the origin, then $D \Phi(0,0)=0$.

Proof. First, we recall that the linear map $D \Phi(0,0): X \times X^{*} \rightarrow Y$ satisfies

$$
\lim _{\left(x, x^{*}\right) \rightarrow(0,0)} \frac{\left\|\Phi\left(x, x^{*}\right)-\Phi(0,0)-D \Phi(0,0)\left(x, x^{*}\right)\right\|}{\left\|\left(x, x^{*}\right)\right\|}=0 .
$$

Then, we claim that $D \Phi(0,0)(x, 0)=0$ for any $x \in K$. To see this, applying (4), we see that for $x \in K \backslash\{0\}$ there holds

$$
0=\lim _{t \rightarrow 0^{+}} \frac{\|\Phi(t x, 0)-\Phi(0,0)-D \Phi(0,0)(t x, 0)\|}{\|t x\|}=\frac{\|D \Phi(0,0)(x, 0)\|}{\|x\|},
$$

which yields $D \Phi(0,0)(x, 0)=0$. Similarly, we can also obtain $D \Phi(0,0)\left(0, x^{*}\right)=0$ for any $x^{*} \in K^{+}$.

Since $K$ and $K^{+}$are solid, by Lemma 2.1, for every $x \in X$ and $x^{*} \in X^{*}$ there exist $x_{1}, x_{2} \in K$ and $x_{1}^{*}, x_{2}^{*} \in K^{+}$such that $x=x_{1}-x_{2}$ and $x^{*}=x_{1}^{*}-x_{2}^{*}$. Hence

$$
\begin{aligned}
& D \Phi(0,0)\left(x, x^{*}\right) \\
= & D \Phi(0,0)\left(x_{1}, 0\right)-D \Phi(0,0)\left(x_{2}, 0\right)+D \Phi(0,0)\left(0, x_{1}^{*}\right)-D \Phi(0,0)\left(0, x_{2}^{*}\right) \\
= & 0
\end{aligned}
$$

which says $D \Phi(0,0)=0$. 
Theorem 2.1. There is no L-pseudoconvex C-function for the GCP.

Proof. Assume that there exists an $L$-pseudoconvex C-function $\Phi: X \times X^{*} \longrightarrow Y$ for the GCP. Taking $x \in \operatorname{int} K$ and $x^{*} \in K^{+} \backslash\{0\}$, applying Lemma 2.4 and the first implication in the definition of $L$-pseudoconvexity, we have $\Phi\left(x, x^{*}\right) \geq_{L} \Phi(0,0)=0$; which, by Lemma 2.3, implies that $\Phi\left(x, x^{*}\right)=0$. Therefore, in view of (3) we must have $\left\langle x, x^{*}\right\rangle=0$, which is a contradiction, since from $x \in \operatorname{int} K$ and $x^{*} \in K^{+} \backslash\{0\}$ it follows that $\left\langle x, x^{*}\right\rangle>0$. Hence, the proof is complete.

\section{Final Remark}

In this paper, we establish that the result done by Miri and Effati's in [21] also holds for general complementarity functions associated with the GCP. Since the GCP includes NCP, SDCP, SOCCP, and SCCP as special cases, this is indeed a nice property for a wide range of complementarity problems.

With this, we point out something in the setting of SOCCP. In such setting, a function $\Phi: \mathbb{R}^{n} \times \mathbb{R}^{n} \rightarrow \mathbb{R}^{n}$ is called a complementarity function (C-function) associated with SOCCP if

$$
\Phi(x, y)=0 \quad \Longleftrightarrow \quad\langle x, y\rangle=0, \quad x \in \mathcal{K}^{n}, y \in \mathcal{K}^{n} .
$$

Here are some $C$-functions associated with SOCCP which are extended from some wellknown and popular NCP-functions via Jordan algebra:

(1) $\Phi_{1}(x, y)=x+y-\left(x^{2}+y^{2}\right)^{1 / 2}$

(2) $\Phi_{3}(x, y)=x-(x-y)_{+}$, (see [14])

(3) $\Phi_{2}(x, y)=x+y-\left(|x|^{p}+|y|^{p}\right)^{1 / p}$, for $p>1$

(4) $\Phi_{4}(x, y)=x^{p}-\left[(x-y)_{+}\right]^{p}$, for $p>1$ being an odd integer (see [19])

(5) $\Phi_{5}(x, y)=\left(\sqrt{x^{2}+y^{2}}\right)^{p}-(x+y)^{p}$, for $p>1$ being an odd integer (see [19])

For more other $C$-functions associated with SOCCP, please refer to $[3,6]$. Note that the second-order cone $\mathcal{K}^{n}$ is self-dual, and it is also a solid closed convex cone. From the main result, we also conclude every $\mathcal{K}^{n}$-convex complementarity function associated with SOCCP is non-differentiable as well. Therefore, several new $C$-functions like $\Phi_{4}, \Phi_{5}$ which are recently proved continuously differentiable in [19], must not be $\mathcal{K}^{n}$-convex.

\section{References}

[1] L. D. Berkovitz, Convexity and Optimization in $\mathbb{R}^{n}$, Wiley-Interscience, New York, 2002. 
[2] J.-S. CHEn, The convex and monotone functions associated with second-order cone, Optimization, vol. 55, no. 4, pp. 363-385, 2006.

[3] J.-S. CHEn, Two classes of merit functions for the second-order cone complementarity problem, Mathematical Methods of Operations Research, vol. 64, pp. 495-519, 2006.

[4] J.-S. Chen, Xin Chen, And P. Tseng, Analysis of nonsmooth vector-valued functions associated with second-order cones, Mathematical Programming, vol. 101, pp. 95-117, 2004.

[5] J.-S. Chen, Xin Chen, S. Pan, And Jiawei Zhang, Some characterizations for SOC-monotone and SOC-convex functions, Journal of Global Optimization, vol. 45, pp. 259-279, 2009.

[6] J.-S. Chen And S.-H. PAn, A survey on SOC complementarity functions and solution methods for SOCPs and SOCCPs, Pacific Journal of Optimization, vol. 8, no. 1, pp. 33-74, 2012.

[7] J.-S. Chen AND P. Tseng An unconstrained smooth minimization reformulation of the second-order cone complementarity problem, Mathematical Programming, vol. 104, pp. 293-327, 2005.

[8] Y. Chiang Characterizations for solidness of dual cones with applications, Journal of Global Optimization, vol. 52. pp. 79-94, 2012.

[9] R. W. Cottle, J.-S. Pang And R.-E. Stone, The Linear Complementarity Problem, Academic Press, New York, 1992.

[10] F. Facchinei And J.-S. PAng, Finite-Dimensional Variational Inequalities and Complementarity Problems, Springer Verlag, New York, 2003.

[11] J. Faraut and A. Korányi, Analysis on Symmetric Cones, Oxford Mathematical Monographs(New York: Oxford University Press), 1994.

[12] A. Galántai, Properties and construction of NCP functions, Computational Optimization and Applications, vol. 52, pp. 805-824, 2012.

[13] P. T. HARKer And J.-S. PANG, Finite dimensional variational inequality and nonlinear complementarity problem: a survey of theory, algorithms and applications, Mathematical Programming, vol. 48, pp. 161-220, 1990.

[14] S. Hayashi, N. Yamashita And M. Fukushima, Robust Nash equilibria and second-order cone complementarity problems, Journal of Nonlinear and Convex Analysis, vol. 6, pp. 283-296, 2005. 
[15] G. IsAC, Topological Methods in Complementarity Theory, Kluwer Academic Publishers, Netherlands, 2000.

[16] Y. Kanno, A. C. Martins and A. Pinto da Costa, Three-dimensional quasistatic frictional contact by using second-order cone linear complementarity problem, International Journal for Numerical Methods in Engineering, vol. 65, pp. 62-83, 2006.

[17] S. Karmardian, Generalized complementarity problem, Journal of Optimization Theory and Applications, vol. 8, pp. 161-168, 1971.

[18] L. C. Kong, L. Tuncel And N. H. Xiu, Vector-valued implicit Lagrangian for symmetric cone complementarity problems, Asia-Pacific Journal of Operational Research, vol. 26, pp. 199-233, 2009.

[19] P. Ma, J.-S. Chen, C.-H. Huang, and C.-H. Ko, Discovery of new complementary functions for NCP and SOCCP, submitted manuscript, 2015.

[20] O. L. Mangasarian, Equivalence of the complementarity problem to a system of nonlinear equations, SIAM Journal on Applied Mathematics, vol. 31, pp. 89-92, 1976.

[21] S. M. Miri AND S. EFFati, On generalized convexity of nonlinear complementarity functions, Journal of Optimization Theory and Applications, vol. 164, pp. 723-730, 2015.

[22] S.-H. PAn AND J.-S. Chen, A one-parametric class of merit functions for the symmetric cone complementarity problem, Journal of Mathematical Analysis and Applications, vol. 355, pp. 195-215, 2009.

[23] S.-H. PAn, S.-G. Kum, Y.-G. Lim And J.-S. Chen,, On the generalized FischerBurmeister merit function for the second-order cone complementarity problem, Mathematics of Computation, vol. 83, pp. 1143-1171, 2014.

[24] D. Sun AND J. Sun, Löwner's operator and spectral functions in Euclidean Jordan algebras, Mathematics of Operations Research, vol. 33, pp. 421-445, 2008. 